\title{
Performance Analysis of Generalized Zero-Padded Blind Channel Estimation Algorithms
}

\author{
Borching Su, Student Member, IEEE, and P. P. Vaidyanathan, Fellow, IEEE
}

\begin{abstract}
In this letter, we analyze the performance of a recently reported generalized blind channel estimation algorithm. The algorithm has a parameter called repetition index, and it reduces to two previously reported special cases when the repetition index is chosen as unity and as the size of received blocks, respectively. The theoretical performance of the generalized algorithm is derived in high-SNR region for any given repetition index. A recently derived Cramer-Rao bound (CRB) is reviewed and used as a benchmark for the performance of the generalized algorithm. Both theory and simulation results suggest that the performance of the generalized algorithm is usually closer to the CRB when the repetition index is larger, but the performance does not achieve the CRB for any repetition index.
\end{abstract}

Index Terms-Blind identification, Cramer-Rao bound, repetition index, zero padding.

\section{INTRODUCTION}

I $\mathrm{N}$ this letter, we analyze the performance of a recently reported blind channel estimation algorithm [5], which is a generalization of the pioneering algorithm advanced in [2] by Scaglione, Giannakis, and Barbarosa (the SGB algorithm). The generalized algorithm in [5] contains a parameter called the repetition index $Q$; when $Q=1$, it reduces to the SGB algorithm. When $Q$ is equal to the size of a received block, the algorithm reduces to the interesting method proposed by Manton, Neumann, and Pham (the MNP algorithm [3], [4]), which allows blind identification with as few as two received blocks. Even though the performance with two blocks is usually not satisfactory, with $Q$ and the number of received blocks adjusted appropriately, the performance of the generalized algorithm in [5] is superior to those of the SGB and MNP algorithms, as documented in detail in [5].

The goal here is to quantify this performance improvement theoretically. We study the channel estimation error (MSE) in the algorithm of [5] and compare it with the corresponding Cramer-Rao bound (CRB). This study is facilitated by the fundamental work reported in [1], which analyzes the SGB algorithm performance, derives appropriate $\mathrm{CRB}$ on the performance, and compares them. The analysis of the generalized algorithm of [5] is included in Section III and follows readily from a modification of the analysis in [1]. We use the CRB

Manuscript received March 20, 2007; revised April 9, 2007. This work was supported in part by the NSF Grant CCF-0428326, in part by ONR Grant N00014-06-1-0011, and in part by the Moore Fellowship of the California Institute of Technology. The associate editor coordinating the review of this manuscript and approving it for publication was Dr. Xiang-Gen Xia.

The authors are with the California Institute of Technology, Pasadena, CA 91125 USA.

Digital Object Identifier 10.1109/LSP.2007.900033 expression given in [6] as a benchmark (this is a corrected version of [1]).

The rest of this letter is organized as follows. Section II briefly gives the problem statement and reviews the generalized algorithm proposed in [5]. In Section III, we derive the theoretical performance of the generalized algorithm and compare it with the CRB. In Section IV, simulation results are given to compare the theoretical performance, performance obtained by simulation, and the CRB. Finally, the conclusion is given in Section V.

\section{A. Notation}

Boldfaced lowercase letters (e.g., v) represent column vectors. Boldfaced uppercase letters and calligraphic uppercase letters are reserved for matrices. Superscripts ${ }^{*},{ }^{T}$, and ${ }^{\dagger}$ as in $\mathbf{A}^{*}$, $\mathbf{A}^{T}$, and $\mathbf{A}^{\dagger}$ denote the conjugate, transpose, and transposeconjugate operations, respectively. $\mathbf{A}^{\#}$ represents the pseudoinverse of $\mathbf{A}$. $[\mathbf{v}]_{k}$ denotes the $k$ th entry of vector $\mathbf{v}$. All the vectors and matrices in this letter are complex-valued. Column and row indices of all matrices and vectors begin at one. $\mathbf{I}_{M}$ is the $M \times M$ identity matrix, and $\mathbf{0}_{m \times n}$ is the $m \times n$ zero matrix. $\mathcal{T}_{k}(\mathbf{v})$ is a $k$-column full-banded Toeplitz matrix [7] whose first column is $\left[\mathbf{v}^{T}, \mathbf{0}_{(k-1)}^{T}\right]^{T}$ and whose last column is $\left[\mathbf{0}_{(k-1)}^{T}, \mathbf{v}^{T}\right]^{T}$. The notation $\operatorname{vec}(\mathbf{A})$ represents the column vector constructed by concatenating columns of $\mathbf{A} . \mathbf{A} \otimes \mathbf{B}$ denotes the Kronecker product [7] of the matrices $\mathbf{A}$ and $\mathbf{B}$.

\section{REVIEW OF THE GENERALIZED ALGORITHM}

\section{A. Problem Formulation}

Consider a sequence of discrete-time information symbols $s(n)$, which is blocked into vectors of size $M$. Let $\mathbf{s}(n)=$ $\left[\begin{array}{llll}s_{0}(n) & s_{1}(n) & \ldots & s_{M-1}(n)\end{array}\right]^{T}$, where $s_{k}(n):=s(M n+k)$ for $k=0,1, \ldots, M-1$. Each block $\mathbf{s}(n)$ is precoded by a linear transformation characterized by an $M \times M$ nonsingular matrix $\mathbf{F}$ so that $\mathbf{u}(n)=\mathbf{F s}(n)$. Each precoded block $\mathbf{u}(n)$ is appended at the end with a block of $L$ zeros, forming a vector $\mathbf{u}_{P}(n)=\left[\begin{array}{ll}\mathbf{u}(n)^{T} & \mathbf{0}_{1 \times L}^{T}\end{array}\right]^{T}$ of size $P=M+L$. The vector signal $\mathbf{u}_{P}(n)$ is unblocked into scalar form $u(n)$ before being sent over the channel. The channel is characterized as a linear time-invariant (LTI) finite impulse response (FIR) system whose order is upper-bounded by $L$ as in the following:

$$
H(z)=\sum_{k=0}^{L} h(k) z^{-k} .
$$

Also define $\mathbf{h}=[h(0) h(1) \ldots h(L)]^{T}$ as the $(L+1)$-vector containing the channel coefficients. The channel output is corrupted by an additive complex white Gaussian noise $e(n)$ with 
variance $\sigma_{v}^{2}$. At the receiver side, the symbol stream $y(n)$ is blocked into vectors of size $P$, which can be written as $\mathbf{y}(n)=$ $\left[\begin{array}{llll}y_{0}(n) & y_{1}(n) & \ldots & y_{P-1}(n)\end{array}\right]^{T}$, where $y_{k}(n):=y(P n+k)$ for $k=0,1, \ldots, P-1$. Assuming the block synchronization between the transmitter and the receiver is perfect, it can be shown that [2]

$$
\mathbf{y}(n)=\mathbf{H F s}(n)+\mathbf{e}(n)
$$

where $\mathbf{H}:=\mathcal{T}_{M}(\mathbf{h})$ is a full-banded Toeplitz matrix and $\mathbf{e}(n)$ is the blocked version of the additive noise $e(n)$. Suppose we collect $J$ received blocks in a $P \times J$ matrix $\mathbf{Y}^{(J)}=\left[\begin{array}{llll}\mathbf{y}(0) & \mathbf{y}(1) & \ldots & \mathbf{y}(J-1)\end{array}\right]$. Then it is clear that

$$
\mathbf{Y}^{(J)}=\mathbf{H U}^{(J)}+\text { noise }
$$

where $\mathbf{U}^{(J)}=\left[\begin{array}{llll}\mathbf{u}(0) & \mathbf{u}(1) & \ldots & \mathbf{u}(J-1)\end{array}\right]$ contains unknown transmitted blocks.

The problem of blind channel estimation can be stated as follows. Given a $P \times J$ matrix $\mathbf{Y}^{(J)}$, how do we estimate the channel coefficients $\mathbf{h}$ blindly (i.e., when $\mathbf{s}(n)$ is unknown)? This problem was first formulated and solved by Scaglione et al. [2]. We will study here the performance of a generalization of the SGB algorithm proposed in [5].

\section{B. Generalized Algorithm}

In this subsection, we review the generalized algorithm proposed in [5]. We start with a subroutine that is used by both SGB algorithm and the generalized algorithm.

Subroutine 1: $(\hat{\mathbf{h}}, \tilde{\mathbf{R}}, \tilde{\mathcal{U}})=\operatorname{ZPBLIND}(\mathbf{Z})$

Input: Matrix $\mathbf{Z}$ of user-defined size $p \times k$.

Outputs: $(L+1)$-vector $\hat{\mathbf{h}}, p \times L$ matrix $\tilde{\mathbf{R}}$, and $(L+1) \times m L$ matrix $\tilde{\mathcal{U}}$, where $m:=p-L$.

1) Take SVD on $\mathbf{Z}$ and denote this as

$$
\mathbf{Z}=\left[\begin{array}{ll}
\overline{\mathbf{R}} & \tilde{\mathbf{R}}
\end{array}\right]\left[\begin{array}{lll}
\boldsymbol{\Sigma}_{s} & & \\
& \boldsymbol{\Sigma}_{n} & \mathbf{0}
\end{array}\right]\left[\begin{array}{c}
\overline{\mathbf{V}}^{\dagger} \\
\tilde{\mathbf{V}}^{\dagger}
\end{array}\right]
$$

where $\boldsymbol{\Sigma}_{n}$ has size $L \times L$ and contains the $L$ smallest singular values of $\mathbf{Z}$. Columns of $\tilde{\mathbf{R}}$, denoted as $\tilde{\mathbf{r}}_{l}$, $l=1,2, \ldots, L$, are the corresponding left singular vectors. (Remark: In particular, if $\mathbf{Z}$ can be written as $\mathbf{Z}=\mathcal{T}_{m}(\mathbf{h}) \mathbf{U}$, where the $m \times k$ matrix $\mathbf{U}$ has rank $m$, then it can be shown that $\boldsymbol{\Sigma}_{n}=\mathbf{0}, \tilde{\mathbf{R}} \mathbf{Z}=\mathbf{0}$, and $\tilde{\mathbf{R}} \mathcal{T}_{m}(\mathbf{h})=\mathbf{0}$, i.e., columns of $\tilde{\mathbf{R}}, \tilde{\mathbf{r}}_{l}, l=1,2, \ldots, L$, are annihilators of $\mathcal{T}_{m}(\mathbf{h})$.)

2) Construct the $(L+1) \times m$ Hankel matrix

$$
\tilde{\mathcal{U}}_{l}:=\left[\begin{array}{cccc}
\tilde{r}_{l}(1) & \tilde{r}_{l}(2) & \ldots & \tilde{r}_{l}(m) \\
\tilde{r}_{l}(2) & \tilde{r}_{l}(3) & \ldots & \tilde{r}_{l}(m+1) \\
\vdots & \vdots & \vdots & \vdots \\
\tilde{r}_{l}(L+1) & \tilde{r}_{l}(L+2) & \ldots & \tilde{r}_{l}(p)
\end{array}\right]
$$

for $l=1,2, \ldots, L$, where $\tilde{r}_{l}(i)$ represents the $i$ th element of $\tilde{\mathbf{r}}_{l}$. Construct matrix $\tilde{\mathcal{U}}:=\left[\begin{array}{llll}\tilde{\mathcal{U}}_{1} & \tilde{\mathcal{U}}_{2} & \ldots & \tilde{\mathcal{U}}_{L}\end{array}\right]$. (Remark: If $\tilde{\mathbf{R}} \mathcal{T}_{m}(\mathbf{h})=\mathbf{0}$, it is readily verified that $\mathbf{h}^{\dagger} \tilde{\mathcal{U}}=\mathbf{0}^{\dagger}$.)

3) Let $\hat{\mathbf{h}}=\arg \min _{\overline{\mathbf{h}}}\|\overline{\mathbf{h}} \dagger \tilde{\mathcal{U}}\|^{2}$.

Subroutine 1 produces an output $\hat{\mathbf{h}}$ proportional to $\mathbf{h}$ (i.e., $\hat{\mathbf{h}}=$ $\alpha \mathbf{h}$ for some $\alpha \in \mathbb{C}$ ) if the input $\mathbf{Z}$ can be written as $\mathbf{Z}=$
$\mathcal{T}_{m}(\mathbf{h}) \mathbf{U}$, where $\mathbf{u}$ has rank $m$. When $\mathbf{Z}$ is corrupted with small additive noise, then columns of $\tilde{\mathbf{R}}$ are approximately annihilators of $\mathcal{T}_{m}(\mathbf{h})$, and an estimate of $\mathbf{h}$ is outputted (with a scalar ambiguity). These properties were first used by Scaglione $e t$ al. in [2] when developing the SGB algorithm. In fact, the SGB algorithm simply runs $(\hat{\mathbf{h}}, \tilde{\mathbf{R}}, \tilde{\mathcal{U}})=\operatorname{ZPBLIND}\left(\mathbf{Y}^{(J)}\right)$ (under the assumption that $\mathbf{U}^{(J)}$ has full rank $M$ ) and takes $\hat{\mathbf{h}}$ as the estimated channel coefficients.

Although the SGB algorithm uses Subroutine 1 as its kernel routine, it does not take advantage of the flexibility on input matrix size of Subroutine 1 (it always uses $p=P$ ). The generalized algorithm in [5], on the other hand, fully exploits this flexibility by using an extra parameter, namely, the repetition index $Q$, as described below.

Algorithm 1: $\hat{\mathbf{h}}=\operatorname{GENERAL}\left(\mathbf{Y}^{(J)}, Q\right)$

Inputs: $P \times J$ matrix $\mathbf{Y}^{(J)}$ and repetition index $Q \geq 1$.

Output: channel estimate as an $(L+1)$-vector $\hat{\mathbf{h}}$.

1) Construct the $(P+Q-1) \times J Q$ matrix

$$
\mathbf{Y}_{Q}^{(J)}=\left[\begin{array}{llll}
\mathcal{T}_{Q}(\mathbf{y}(0)) & \mathcal{T}_{Q}(\mathbf{y}(1)) & \ldots & \mathcal{T}_{Q}(\mathbf{y}(J-1))
\end{array}\right] .
$$

2) Perform the subroutine $(\hat{\mathbf{h}}, \tilde{\mathbf{R}}, \tilde{\mathcal{U}})=\operatorname{ZPBLIND}\left(\mathbf{Y}_{Q}^{(J)}\right)$ and output $\hat{\mathbf{h}}$.

The generalized algorithm is based on the idea that (1) implies

$$
\mathbf{Y}_{Q}^{(J)}=\mathcal{T}_{M+Q-1}(\mathbf{h}) \mathbf{U}_{Q}^{(J)}+\text { noise }
$$

where

$$
\mathbf{U}_{Q}^{(J)}=\left[\begin{array}{llll}
\mathcal{T}_{Q}(\mathbf{u}(0)) & \mathcal{T}_{Q}(\mathbf{u}(1)) & \ldots & \mathcal{T}_{Q}(\mathbf{u}(J-1))
\end{array}\right] .
$$

Note that the noise autocorrelation in (4) is different from that in (1). When $Q=1$, the generalized algorithm reduces to the SGB algorithm. Also, when $Q=P$, the generalized algorithm is equivalent to the MNP algorithm [4]. The matrix $\mathbf{U}_{Q}^{(J)}$ must have full rank so that Algorithm 1 works, which implies $J \geq$ $1+\lceil(M-1) / Q\rceil$.

\section{Performance AnAlysis And the Cramer-RaO Bound}

\section{A. Performance Analysis in Additive Noise}

When evaluating the MSE performance of blind estimation algorithms, it is natural to compare the estimated channel $\hat{\mathbf{h}}$ and the true channel $\mathbf{h}$. However, due to an intrinsic scalar ambiguity presented in all blind channel estimation algorithms, the comparison should be done after normalizing this unknown scalar. There are many options for doing this. Here we adopted an option presented in [1], where the channel coefficient with the largest magnitude is assumed known. That is, $h(d)$, where $d \in[0, L]$ satisfies $|h(d)| \geq|h(l)|, \forall l \neq d$, is known. After normalizing the estimated channel vector by letting $\tilde{\mathbf{h}}=(h(d) / \hat{h}(d)) \hat{\mathbf{h}}$, the channel estimation error can be defined as an $L$-vector

$$
\Delta \mathbf{h}=\mathbf{I}_{L, d}(\tilde{\mathbf{h}}-\mathbf{h})
$$

where $\mathbf{I}_{L, d}$ is an $L \times(L+1)$ matrix obtained by removing the $d$ th row of $\mathbf{I}_{L+1}$. We first review a result on estimating $\Delta \mathbf{h}$ presented 
in [1] using small perturbation analysis. In Lemma 1 presented below, we assume the perturbation $\Delta \mathbf{X}$ is small compared to $\mathbf{X}$. That is, assume the first-order approximation

$$
(\mathbf{X}+\Delta \mathbf{X})^{\dagger}(\mathbf{X}+\Delta \mathbf{X}) \approx \mathbf{X}^{\dagger} \mathbf{X}+\Delta \mathbf{X}^{\dagger} \mathbf{X}+\mathbf{X}^{\dagger} \Delta \mathbf{X}
$$

is accurate. We also assume $h(d)$ is known to the receiver and the output of ZPBLIND, $\hat{\mathbf{h}}$ has applied the scalar ambiguity normalization based on the knowledge of $h(d)$.

Lemma 1: Let $\mathbf{X}=\mathcal{T}_{m}(\mathbf{h}) \mathbf{U}$, where $m \times k$ matrix $\mathbf{U}$ has rank $m$. Let $\mathbf{Y}=\mathbf{X}+\Delta \mathbf{X}$, where $\Delta \mathbf{X}$ is a small perturbation to $\mathbf{X}$. Perform subroutine ZPBLIND on $\mathbf{X}$ and $\mathbf{Y}$ and denote them as $\left(\hat{\mathbf{h}}, \mathbf{R}_{1}, \mathcal{U}_{1}\right)=\operatorname{ZPBLIND}(\mathbf{Y})$ and $\left(\mathbf{h}_{1}, \tilde{\mathbf{R}}, \hat{\mathcal{U}}\right)=$ $\mathrm{ZPBLIND}(\mathbf{X})$, respectively. Consider error vector $\Delta \mathbf{h}$ as defined in (6). Then the first-order approximation of $\Delta \mathbf{h}$ can be expressed as

$$
\Delta \mathbf{h}^{\dagger} \approx \operatorname{vec}^{T}\left(\Delta \mathbf{X}^{\dagger}\right)\left(\tilde{\mathbf{R}} \otimes \mathbf{U}^{\# *}\right) \tilde{\mathcal{V}}^{\#}
$$

where $\tilde{\mathcal{V}}:=\mathbf{I}_{L, d} \tilde{\mathcal{U}}$.

Proof: See [1, Eq. (28)].

Notice that $\tilde{\mathbf{R}}$ and $\tilde{\mathcal{V}}$ depend only on $\mathbf{U}$ and $\mathbf{h}$ and not on the noise perturbation $\Delta \mathbf{X}$. Using Lemma 1 , we can derive the MSE performance of the generalized algorithm in [5] by computing the autocorrelation matrix of $\Delta \mathbf{h}$, as described below.

Theorem 1: Consider $\mathbf{Y}^{(J)}$ as defined in (1) and $Q$ as the repetition index. Perform Algorithm 1: $\hat{\mathbf{h}}=$ $\operatorname{GENERAL}\left(\mathbf{Y}^{(J)}, Q\right)$. Then the autocorrelation matrix of the channel estimation error vector $\Delta \mathbf{h}$ [defined in (6)] can be expressed as

$$
\begin{aligned}
\mathbf{C}_{h h, Q}= & E\left[\Delta \mathbf{h} \Delta \mathbf{h}^{\dagger}\right] \\
\approx & \sigma_{v}^{2} \tilde{\mathcal{V}}_{Q}^{\# \dagger}\left(\tilde{\mathbf{R}}_{Q}^{\dagger} \otimes \mathbf{U}_{Q}^{(J)} \#_{T}\right) \\
& \times\left(\mathbf{I}_{J} \otimes \mathbf{B}_{Q}\right)\left(\tilde{\mathbf{R}}_{Q} \otimes \mathbf{U}_{Q}^{(J) \# *}\right) \tilde{\mathcal{V}}_{Q}^{\#} .
\end{aligned}
$$

Here $\mathbf{U}_{Q}^{(J)}$ is defined as in (5), and $\mathbf{B}_{Q}$ is defined as

$$
\begin{aligned}
\mathbf{B}_{Q} & =\left[\begin{array}{ccc}
\mathbf{B}_{11} & \ldots & \mathbf{B}_{1 Q} \\
\vdots & \vdots & \vdots \\
\mathbf{B}_{Q 1} & \ldots & \mathbf{B}_{Q Q}
\end{array}\right] \\
\mathbf{B}_{k l} & =\left[\begin{array}{c}
\mathbf{0}_{(k-1) \times(P+Q-1)} \\
\mathbf{K}_{l} \\
\mathbf{0}_{(Q-k) \times(P+Q-1)}
\end{array}\right] \\
\mathbf{K}_{l} & =\left[\begin{array}{l}
\mathbf{0}_{(P+Q-1) \times(l-1)} \\
\mathbf{I}_{P}
\end{array} \mathbf{0}_{(P+Q-1) \times(Q-l)}\right] .
\end{aligned}
$$

In (7), $\tilde{\mathbf{R}}_{Q}$ and $\tilde{\mathcal{V}}_{Q}$ are obtained by performing

$$
\left(\hat{\mathbf{h}}^{\prime}, \tilde{\mathbf{R}}_{Q}, \tilde{\mathcal{U}}_{Q}\right)=\operatorname{ZPBLIND}\left(\mathcal{T}_{M+Q-1}(\mathbf{h}) \mathbf{U}_{Q}^{(J)}\right)
$$

and letting $\tilde{\mathcal{V}}_{Q}=\mathbf{I}_{L, d} \tilde{\mathcal{U}}_{Q}$.

Proof: Using Lemma 1, the autocorrelation matrix of $\Delta \mathbf{h}$ can be written as

$$
E\left[\Delta \mathbf{h} \Delta \mathbf{h}^{\dagger}\right]=\tilde{\mathcal{V}}^{\# \dagger}\left(\tilde{\mathbf{R}}^{\dagger} \otimes \mathbf{U}^{\# T}\right) \mathbf{R}_{\Delta \mathbf{X}}\left(\tilde{\mathbf{R}} \otimes \mathbf{U}^{\# *}\right) \tilde{\mathcal{V}}^{\#}
$$

where $\mathbf{R}_{\Delta \mathbf{X}}:=E\left[\operatorname{vec}\left(\Delta \mathbf{X}_{Q}^{(J)}\right) \operatorname{vec}^{\dagger}\left(\Delta \mathbf{X}_{Q}^{(J)}\right)\right]$. The perturbation matrix $\Delta \mathbf{X}_{Q}^{(J)}$ can be written as

$$
\Delta \mathbf{X}_{Q}^{(J)}=\left[\begin{array}{llll}
\mathcal{T}_{Q}(\mathbf{e}(0)) & \mathcal{T}_{Q}(\mathbf{e}(1)) & \ldots & \mathcal{T}_{Q}(\mathbf{e}(J-1))
\end{array}\right]
$$

One can verify that the $(P+Q-1) J Q \times(P+Q-1) J Q$ matrix $\mathbf{R}_{\Delta \mathbf{X}}$ can be written as $\mathbf{R}_{\Delta \mathbf{X}}=\sigma_{v}^{2}\left(\mathbf{I}_{J} \otimes \mathbf{B}_{Q}\right)$, and the proof of the theorem is complete.

Corollary 1: When $Q=1$, the channel estimation error autocorrelation matrix can be expressed as

$$
\mathbf{C}_{h h, 1}=\sigma_{v}^{2} \tilde{\mathcal{V}}^{\# \dagger}\left(\mathbf{I}_{L} \otimes \mathbf{U}^{(J) \# T} \mathbf{U}^{(J) \# *}\right) \tilde{\mathcal{V}}^{\#}
$$

which agrees with the analytical performance of the SGB algorithm derived in [1].

Proof: Immediate from Theorem 1 using $\mathbf{B}_{Q}=\mathbf{I}_{P}$ and the fact that columns of $\tilde{\mathbf{R}}_{Q}$ are orthogonal to each other.

\section{B. Cramer-Rao Bound}

In [1], a CRB for the zero-padding blind channel estimation problem was derived. We use the corrected version CRB presented in [6] as follows:

$$
\mathbf{C}_{\mathrm{CR}}=\sigma_{v}^{2}\left[\tilde{\mathcal{V}}\left(\mathbf{I}_{L \times L} \otimes \mathbf{U}^{(J) *} \mathbf{U}^{(J) T}\right) \tilde{\mathcal{V}}^{\dagger}\right]^{-1} .
$$

The Cramer-Rao bound presented in (9) is a lower bound for the performance of all algorithms that attempt to solve the blind estimation problem described in Section II-A, including the SGB algorithm [2], the MNP algorithm [4], and the generalized algorithm [5].

\section{Simulations}

In this section, we perform Monte Carlo simulation for the generalized blind channel estimation algorithm [5] with different repetition indices and compare the performance obtained by simulations and theory as well as the Cramer-Rao bound at different SNR values.

In our simulations, the block size is chosen as $M=12$ and the channel order is chosen as $L=4$. The QPSK constellation is used to generate i.i.d. symbols $s(n)$, and the linear precoder $\mathbf{F}$ is chosen as $\mathbf{I}_{M}$. The channel coefficients (elements of $\mathbf{h}$ ) are chosen as i.i.d., zero-mean, unit variance complex Gaussian random variables. The simulation is performed using 100 independent realizations of channel coefficients and ten independent realizations of symbol streams $s(n)$ (totally 1000 different pairs of $\mathbf{S}^{(J)}$ and $\mathbf{h}$ ). Theoretical performances in (7) and the CRB in (9) are computed accordingly and averaged over these 1000 pairs of $\mathbf{S}^{(J)}$ and $\mathbf{h}$. Furthermore, to solve the scalar ambiguity problem, the channel coefficient with the largest magnitude, $h(d)$, is assumed known to the receiver. Two separate simulation settings are considered: the first one uses 16 received blocks $(J=16)$ and the second one uses $J=5$.

Fig. 1 depicts the result of the first simulation setting, where $J=16$. We compare the MSE performances with $Q=1$ and $Q=2$. Both theoretical and simulation performances are plotted for each case. The CRB is plotted as the benchmark. We have the following observations. First of all, in both cases 


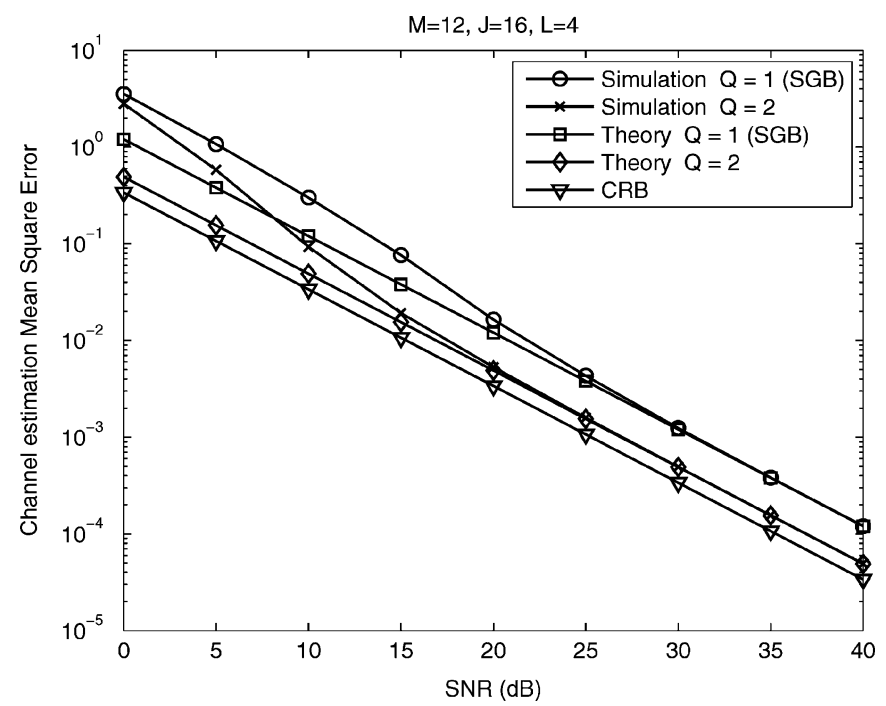

Fig. 1. Channel estimation MSE versus SNR obtained by simulations, theoretical values in (7), and CRB in (9) with 16 blocks.

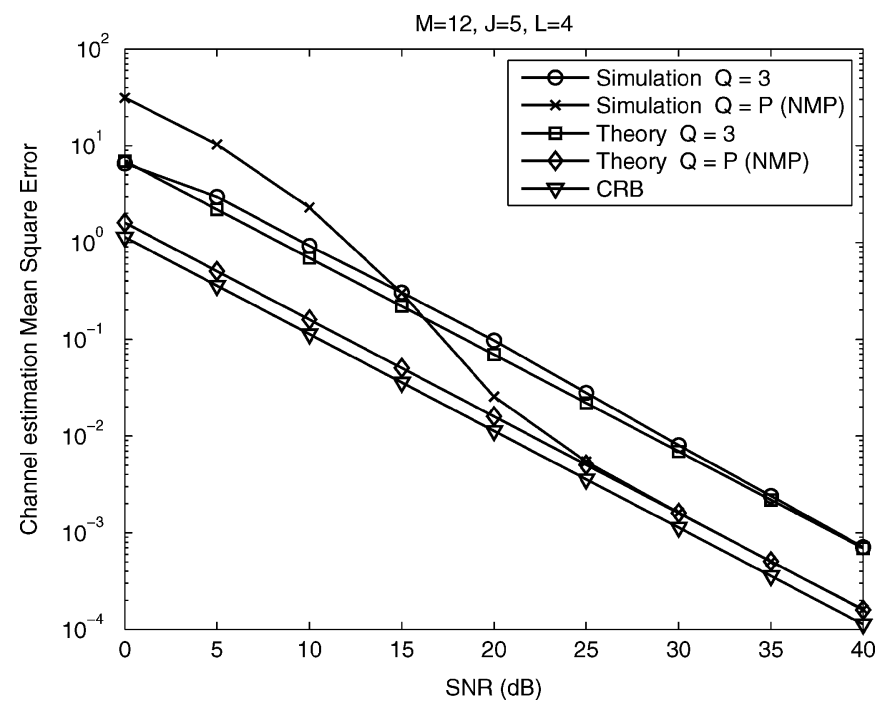

Fig. 2. Channel estimation MSE versus SNR obtained by simulations, theoretical values in (7), and CRB in (9) with five blocks.

of $Q=1$ and $Q=2$, the simulation results are very close to theory in the high SNR region. This validates the small perturbation assumption given in Lemma 1. Second, performance of $Q=2$ is better than that of $Q=1$ with a considerable margin. However, the system with $Q=2$ does not achieve the CRB yet.
Increasing $Q$ might further improve the performance toward the CRB, but we omit these curves here due to space limit.

In Fig. 2, simulation results are shown for the case when $J=$ 5. We choose $Q=3$ and $Q=P(=M+L=16)$ in this simulation. Notice that $Q=P$ represents the MNP algorithm [4]. The simulation results approach the theoretical values when SNR goes to infinity. In high SNR region, the performance for $Q=P$ is obviously better than that for $Q=3$, but it still does not achieve the CRB. Notice that in this case, we need $Q \geq 3$ in order to satisfy the full-rank assumption as described in Section II-B.

\section{CONCLUSION}

In this letter, we derived the theoretical performance of the generalized blind channel estimation algorithm [5] in the high-SNR range. Simulation results and theory both suggest that when the repetition index is larger, the performance is usually better when SNR is large. A CRB presented in [1] and corrected in [6] is used as a benchmark of the algorithm performance. When the repetition index $Q$ is large, the performance curve tends to approach the CRB but does not appear to achieve it.

In the future, a formal proof that the generalized algorithm does not achieve the CRB for any $Q$ is desirable. It also remains an open question whether there exists another blind channel estimation algorithm that has a performance achieving the CRB.

\section{REFERENCES}

[1] S. Barbarossa, A. Scaglione, and G. B. Giannakis, "Performance analysis of a deterministic channel estimator for block transmission systems with null guard intervals," IEEE Trans. Signal Process., vol. 50, no. 3, pp. 684-695, Mar. 2002.

[2] A. Scaglione, G. B. Giannakis, and S. Barbarossa, "Redundant filter bank precoders and equalizers Part II: Synchronization and direct equalization," IEEE Trans. Signal Process., vol. 47, no. 7, pp. 2007-2002, Jul. 1999.

[3] J. H. Manton and W. D. Neumann, "Totally blind channel identification by exploiting guard intervals," Syst. Control Lett., vol. 48, no. 2, pp. 113-119, 2003.

[4] D. H. Pham and J. H. Manton, "A subspace algorithm for guard interval based channel identification and source recovery requiring just two received blocks," in Proc. ICASSP, Hong Kong, China, 2003, pp. 317-320.

[5] B. Su and P. P. Vaidyanathan, "A generalized algorithm for blind channel identification with linear redundant precoders," EURASIP J. Appl. Signal Process., vol. 2007, Article ID 25672, 13 pp.

[6] B. Su and P. P. Vaidyanathan, "Comments on "performance analysis of a deterministic channel estimator for block transmission systems with null guard intervals'," IEEE Trans. Signal Process., submitted for publication.

[7] R. A. Horn and C. R. Johnson, Matrix Analysis. Cambridge, U.K.: Cambridge Univ. Press, 1996. 